\title{
Factores asociados a la no-adherencia al tratamiento farmacológico antihipertensivo en pacientes de un hospital del seguro social
}

\section{Factors associated with non-adherence to antihypertensive treatment in patients with social security}

\section{RESUMEN}

Introducción: La falta de adherencia a la terapia antihipertensiva contribuye directamente a que los pacientes coexistan con hipertensión, desencadenando mayor riesgo de morbilidad y mortalidad. Así, la falta de adherencia al tratamiento se convierte en una de las principales causas de hipertensión no controlada en la población. Evaluamos los factores asociados a la no-adherencia al tratamiento antihipertensivo en pacientes de cardiología de un hospital de EsSalud en San Juan de Lurigancho-Lima, durante el 2017. Material y Métodos: Estudio analítico-transversal, se incluyeron pacientes que acudieron a consultorio externo de cardiología con historia previa de hipertensión esencial y se excluyeron hipertensión secundaria por otras causas biológicas. Se utilizó el Test de Morisky-Green Morisky Medication Adherence Scale (MMAS-4) para evaluar la no-adherencia al tratamiento antihipertensivo. Resultados: De los 270 participantes de estudio, el $69 \%(n=185)$ eran adultos mayores de 65 años, $46 \%$ $(n=124)$ varones y el $76 \%(n=118)$ tenían pareja estable. Entre los antecedentes clínicos, el $60 \%(n=122)$ reportó diagnóstico clínico de hipertensión arterial, el $30 \%(n=80)$ de diabetes mellitus tipo II y el $27 \%(n=73)$ no-adherencia al tratamiento. Los factores asociados a la no-adherencia de tratamiento fueron sexo masculino (ORa: 0,45, IC 95\% 0,20-1,04), trabajador independiente (ORa:3,88, IC95\% 1,51-9,97), IMC mayor de 30 (ORa:0,23, IC95\% 0,07-0,70). Conclusiones: en los pacientes con diagnóstico de hipertensión esencial existen factores de riesgo modificables y no modificables asociados a la no-adherencia al tratamiento. Se deben considerar estos factores para implementar estrategias de tamizaje y focalizar las intervenciones para adherir a los pacientes renuentes a su tratamiento.

Palabras Clave: Tratamiento farmacológico; hipertensión; Cumplimiento y Adherencia al Tratamiento; Antihipertensivos; Servicio de Cardiología en Hospital; Perú (Fuente: DeCS-BIREME).

\section{ABSTRACT}

Background: Lack of adherence to antihypertensive therapy contributes directly to patients coexisting with hypertension,

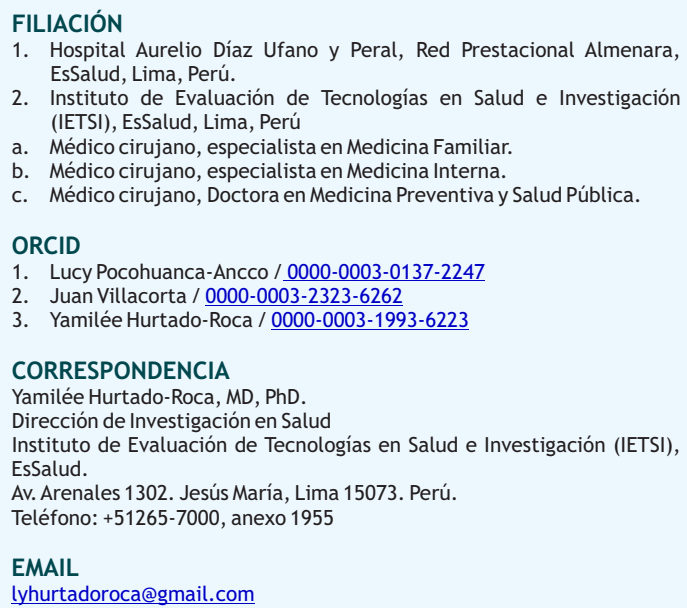

\section{FINANCIAMIENTO}

Esta investigación fue financiada por el Instituto de Evaluación de Tecnologías en Salud e Investigación (IETSI), EsSalud.

\section{CONTRIBUCIONES}

JV, LPA y YHR formularon la idea de investigación. LPA se encargó de la recolección de datos, YHR hizo el análisis de datos. JV, LPA, YHR redactaron el manuscrito y aprobaron la versión final.

\section{AGRADECIMIENTOS}

Los autores agradecemos a la Daphne Solier por su ayuda inicial en la recolección de datos.

\section{REVISIÓN DE PARES}

Recibido: 02/05/2021

Aceptado: $30 / 08 / 202$

\section{COMOCITAR}

Pocohuanca-Ancco, L., Villacorta, J., \& Hurtado-Roca, Y. Factores asociados a la no-adherencia al tratamiento farmacológico antihipertensivo en pacientes de un hospital del seguro social. Revista Del Cuerpo Médico Hospital Nacional Almanzor Aguinaga Asenjo, 2021, 14(3). https://doi.org/10.35434/rcmhnaaa.2021.143.1252

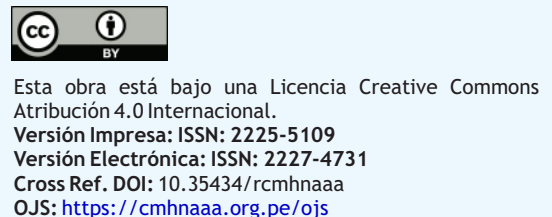


triggering increased risk of morbidity and mortality. Thus, nonadherence to treatment becomes one of the main causes of uncontrolled hypertension in the population. We evaluated the factors associated with non-adherence to antihypertensive treatment in cardiology patients of an EsSalud hospital in San Juan de Lurigancho-Lima, during 2017. Material and Methods: Analytical-crossover study, patients attending cardiology outpatient clinic with previous history of essential hypertension were included and secondary hypertension due to other biological causes were excluded. The Morisky-Green Morisky Medication Adherence Scale (MMAS-4) was used to assess non-adherence to antihypertensive treatment. Results: Of the 270 study participants, $69 \%(n=185)$ were adults older than 65 years, $46 \%(n=124)$ were male and $76 \%(n=118)$ had a stable partner. Among the clinical history, $60 \%(n=122)$ reported clinical diagnosis of arterial hypertension, $30 \%(n=80)$ of type II diabetes mellitus and $27 \%(n=73)$ non-adherence to treatment. Factors associated with non-adherence to treatment were male sex (ORa: $0.45,95 \% \mathrm{Cl} 0.20-1.04)$, self-employed (ORa:3.88, 95\% Cl 1.51-9.97), BMI greater than 30 (ORa:0.23, $95 \% \mathrm{Cl}$ 0.07-0.70). Conclusions: in patients with a diagnosis of essential hypertension there are modifiable and nonmodifiable risk factors associated with non-adherence to treatment. These factors should be considered in order to implement screening strategies and target interventions to adhere to treatment in reluctant patients.

Keywords: Drug Therapy; hypertension, Treatment Adherence and Compliance; Antihypertensive Agents; Cardiology Service, Hospital; Peru. (Source: DeCS-BIREME).

\section{INTRODUCCION}

La falta de adherencia al tratamiento farmacológico es frecuente, sobre todo en condiciones crónicas y está asociada a múltiples factores ${ }^{(1)}$. A pesar que la hipertensión arterial es una de las patologías que cuenta con una vasta opción de tratamiento efectivo, la falta de adherencia puede resultar una barrera para el óptimo control de los pacientes hipertensos. En países desarrollados, tanto en Europa como en Canadá y los Estados Unidos, se está evidenciando un alto porcentaje de pacientes hipertensos no controlados ${ }^{(2)}$; esta falla al tratamiento se describe fundamentalmente por la falta de adherencia a la terapia farmacológica(3). Adicionalmente, se ha reportado que en pacientes hipertensos es frecuente que no se cumpla adecuadamente el tratamiento, registrándose incluso casos de hipertensión refractaria al tratamiento que en realidad corresponden a una falta de adherencia al tratamiento ${ }^{(4)}$. La falta de adherencia a la terapia antihipertensiva contribuye directamente a que los pacientes coexistan con hipertensión, la misma que desencadena eventos que ponen en riesgo de mayores morbilidades y mortalidad a estos pacientes ${ }^{(5-7)}$. Así, la falta de adherencia al tratamiento se convierte en una de las principales causas de hipertensión no controlada en la población ${ }^{(8)}$. Identificar estos factores de riesgo resulta importante para realizar intervenciones efectivas y específicas. La literatura sugiere que la falta de adherencia está más asociada a pacientes jóvenes, con pocas comorbilidades, con algún problema de salud mental, abuso de drogas, de sexo masculino, al tipo de tratamiento farmacológico y tolerabilidad a los eventos adversos ${ }^{(9-14)}$. Sin embargo, estos factores pueden variar en función a los sistemas de salud, cobertura sanitaria e incluso el ingreso económico ${ }^{(15)}$.

En este estudio estimamos la frecuencia de factores asociados a la no-adherencia a la medicación antihipertensiva. Para este fin analizamos los datos de los registros clínicos de pacientes a quienes se les aplicó la escala de Morisky en el Hospital Aurelio Díaz Ufano y Peral.

\section{MATERIALES Y MÉTODOS}

Se realizó un estudio analítico-transversal a partir de los registros médicos y de enfermería para evaluar los factores asociados a la no-adherencia al tratamiento antihipertensivo en pacientes de cardiología del Hospital Aurelio Díaz Ufano y Peral, de la Red Prestacional Almenara, ubicado en San Juan de Lurigancho-Lima, durante el 2017. Se incluyeron los datos de pacientes que acudieron a consultorio externo con historia previa de hipertensión esencial y diabetes mellitus tipo 2 . Se utilizó el registro del Test de Morisky-Green Morisky Medication Adherence Scale (MMAS-4) para evaluar la noadherencia al tratamiento aplicado a los pacientes ambulatorios. Esta escala ha sido validada en pacientes peruanos con diagnóstico de hipertensión arterial esencial ${ }^{(8)}$. Las variables categóricas fueron descritas con frecuencias relativas y las continuas con media y desviación estándar o mediana y rangos intercuartílicos, según correspondía. Se aplicó el estadístico chi-cuadrado para evaluar diferencias entre subgrupos independientes de estudio. Se estimaron los modelos de regresión de la familia de distribución Poisson con enlace log para calcular las razones de prevalencia, con un intervalo de confianza del $95 \%$. Este estudio contó con aprobación del Comité Institucional de Ética en Investigación del Hospital I Aurelio Díaz Ufano y Peral de la Red Desconcentrada Almenara de EsSalud y autorización institucional.

\section{RESULTADOS}

De los 270 participantes de estudio, el 69\% ( $n=185)$ eran adultos mayores de 65 años, 46\% $(n=124)$ varones, el $76 \%$ $(n=118)$ tenían pareja estable. Entre los antecedentes clínicos, el $5 \%(n=14)$ tuvo diagnóstico clínico de síndrome depresivo, el $45 \%(n=122)$ hipertensión arterial, el 25\% $(n=68)$ diabetes mellitus tipo II y $30 \%(n=80)$ registró DM tipo II e hipertensión al mismo tiempo. Sólo el $27 \%$ no estuvo adherido al tratamiento. Los pacientes con mayor adherencia al tratamiento farmacológico fueron pacientes mayores de 65 años y de sexo masculino, el $78 \%(n=95)$ fueron pacientes 
Tabla 1. Características demográficas y clínicas de pacientes con hipertensión y diabetes mellitus tipo 2.

\begin{tabular}{|c|c|c|c|c|c|c|c|}
\hline & & & & herencia & & & \\
\hline & & & Adherido & & No-adherido & & \\
\hline Edad & & & & & & & 0.01 \\
\hline$<65$ & 85 & 31,5 & 53 & 62,35 & 32 & 37,65 & \\
\hline$>=65$ & 185 & 68,5 & 144 & 77,84 & 41 & 22,16 & \\
\hline Sexo & & & & & & & 0,04 \\
\hline Femenino & 146 & 54,1 & 99 & 67,81 & 47 & 32,19 & \\
\hline Masculino & 124 & 45,9 & 98 & 79,03 & 26 & 20,97 & \\
\hline Pareja & & & & & & & 0,31 \\
\hline Con pareja & 118 & 75,2 & 84 & 71,19 & 34 & 28,81 & \\
\hline Sin pareja & 39 & 24,8 & 31 & 79,49 & 8 & 20,51 & \\
\hline Educación & & & & & & & 0,06 \\
\hline Primaria & 28 & 31,5 & 25 & 89,29 & 3 & 10,71 & \\
\hline Secundaria & 45 & 50,6 & 29 & 64,44 & 16 & 35,56 & \\
\hline Superior & 16 & 18 & 12 & 75 & 4 & 25 & \\
\hline Empleo & & & & & & & 0,48 \\
\hline Independiente & 10 & 8,5 & 6 & 60 & 4 & 40 & \\
\hline Empleado & 10 & 8,5 & 6 & 60 & 4 & 40 & \\
\hline Obrero & 6 & 5,1 & 5 & 83,33 & 1 & 16,67 & \\
\hline Otros & 92 & 78 & 70 & 76,09 & 22 & 23,91 & \\
\hline Depresión & 14 & 5,2 & 8 & 57,14 & 6 & 42,86 & 0,17 \\
\hline Hipertensión (HTA) & 122 & 45,2 & 95 & 77,87 & 27 & 22,13 & 0,26 \\
\hline Diabetes mellitus tipo II (DM) & 68 & 25,2 & 45 & 66,18 & 23 & 33,82 & 0,54 \\
\hline$H T A+D M$ & 80 & 29,6 & 57 & 71,25 & 23 & 28,75 & 0,68 \\
\hline Peso (Kg) & $67[43,5-134]$ & & $67,3[43,5-105]$ & & $65,5[47,5-134]$ & & 0,37 \\
\hline Talla (m) & $1,5 \pm 0,1$ & & $1,5 \pm 0,1$ & & $1,6 \pm 0,1$ & & 0,34 \\
\hline Índice de masa corporal & $27,9[19,9-44,6]$ & & $28[19,9-44,3]$ & & $27,4[20,2-44,6]$ & & 0,46 \\
\hline Presión arterial sistólica & $120[100-160]$ & & $120[110-160]$ & & $120[100-150]$ & & 0,96 \\
\hline Presión arterial diastólica & $26[25,7-27,9]$ & & $80[60-90]$ & & $70[60-80]$ & & 0,4 \\
\hline Presión arterial media & $80[60-90]$ & & $106,7[93,3-136,7]$ & & $106,7[90-126,7]$ & & 0,72 \\
\hline Glucosa (mg/dl) & $113[51-396]$ & & $112[74-336]$ & & $119[51-396]$ & & 0,01 \\
\hline Triglicéridos (mg/dl) & 155 [24-747] & & $153[24-564]$ & & $161[52-747]$ & & 0,24 \\
\hline LDL (mg/dl) & $102[8-235]$ & & $100[8-208,7]$ & & $108,5[34,8-235]$ & & 0,3 \\
\hline Adherencia al tratamiento & & & & & & & \\
\hline Adherido & 197 & 73 & & & & & \\
\hline No-adherido & 73 & 27 & & & & & \\
\hline
\end{tabular}

hipertensos, el $66 \%$ diabéticos tipo II y el $71 \%$ tuvo ambas condiciones. Los pacientes que no se adhieren al tratamiento presentaron niveles de glucosa y LDL por encima de los valores normales y en mayor promedio que los pacientes que logran adherirse al tratamiento (Tabla 1). 
Tabla 2. Factores asociados a la no-adherencia al tratamiento antihipertensivo en pacientes con hipertensión y diabetes mellitus tipo II.

\begin{tabular}{|c|c|c|c|c|c|c|c|c|}
\hline & \multicolumn{7}{|c|}{ No-adherencia al tratamiento } & \multirow{2}{*}{ Valor $\mathrm{P}$} \\
\hline & RPc & IC95\% & & Valor $\mathrm{P}$ & $\mathrm{RPa}$ & IC95\% & & \\
\hline \multicolumn{9}{|l|}{ Edad } \\
\hline$<65$ & Ref. & & & & & & & \\
\hline$>=65$ & 0.59 & 0,4 & 0,87 & 0,01 & 0,89 & 0,42 & 1,88 & 0,75 \\
\hline \multicolumn{9}{|l|}{ Sexo } \\
\hline Femenino & Ref. & & & & & & & \\
\hline Masculino & 0.65 & 0,43 & 0,99 & 0,04 & 0,45 & 0,2 & 1,04 & 0,06 \\
\hline \multicolumn{9}{|l|}{ Pareja } \\
\hline Con pareja & Ref. & & & & & & & \\
\hline Sin pareja & 0.71 & 0,36 & 1,41 & 0,33 & 0,63 & 0,25 & 1,58 & 0,33 \\
\hline \multicolumn{9}{|l|}{ Educación } \\
\hline Secundaria & 1.42 & 0,56 & 3,64 & 0,46 & 0,77 & 0,3 & 2 & 0,6 \\
\hline Superior & Ref. & & & & & & & \\
\hline \multicolumn{9}{|l|}{ Empleo } \\
\hline Independiente & 1.67 & 0,72 & 3,9 & 0,23 & 3,88 & 1,51 & 9,97 & 0,01 \\
\hline Empleado & 1.67 & 0,72 & 3,9 & 0,23 & 1,31 & 0,53 & 3,22 & 0,56 \\
\hline Obrero & 0.7 & 0,11 & 4,36 & 0,7 & 1,16 & 0,23 & 5,95 & 0,86 \\
\hline Otros & Ref. & & & & & & & \\
\hline \multicolumn{9}{|l|}{ Depresión } \\
\hline No & Ref. & & & & & & & \\
\hline $\mathrm{Si}$ & 1.64 & 0,86 & 3,11 & 0,13 & 0,8 & 0,13 & 4,86 & 0,81 \\
\hline \multicolumn{9}{|c|}{ Hipertensión (HTA) } \\
\hline No & Ref. & & & & & & & \\
\hline \multicolumn{9}{|c|}{ Diabetes mellitus tipo II (DM) } \\
\hline No & Ref. & & & & & & & \\
\hline $\mathrm{Si}$ & 1.16 & 0,72 & 1,88 & 0,54 & 1,55 & 0,52 & 4,59 & 0,43 \\
\hline \multicolumn{9}{|c|}{ Índice de masa corporal } \\
\hline$<30$ & Ref. & & & & & & & \\
\hline$>=30$ & 0.84 & 0,57 & 1,25 & 0,39 & 0,23 & 0,07 & 0,7 & 0,01 \\
\hline \multicolumn{9}{|c|}{ Presión arterial media (mmHg) } \\
\hline$<105$ & Ref. & & & & & & & \\
\hline$>=105$ & 0.85 & 0,55 & 1,32 & 0,46 & 1,56 & 0,74 & 3,28 & 0,24 \\
\hline \multicolumn{9}{|l|}{ Glucosa (mg/dl) } \\
\hline$<200$ & Ref. & & & & & & & \\
\hline$>=200$ & 1.92 & 1,29 & 2,85 & 0 & 1,59 & 0,67 & 3,79 & 0,29 \\
\hline \multicolumn{9}{|c|}{ Triglicéridos (mg/dl) } \\
\hline$<200$ & Ref. & & & & & & & \\
\hline$>=200$ & 1 & 1 & 1 & 0,14 & 1,77 & 0,88 & 3,57 & 0,11 \\
\hline \multicolumn{9}{|l|}{ LDL (mg/dl) } \\
\hline$<110$ & Ref. & & & & & & & \\
\hline$>=110$ & 1.34 & 0,89 & 2,02 & 0,15 & 1,35 & 0,67 & 2,72 & 0,4 \\
\hline
\end{tabular}

Los factores asociados a la no-adherencia de tratamiento fueron sexo masculino (ORa: 0,45, IC $95 \%$ 0,20-1,04), trabajador independiente (ORa:3,88, IC95\% 1,51-9,97), IMC mayor de 30 (ORa:0,23, IC95\% 0,07-0,70) (Tabla 2). 


\section{DISCUSIÓN}

La hipertensión es un factor de riesgo modificable y cuenta con diversas alternativas farmacológicas para su control. Estudios clínicos aleatorizados han evidenciado que el tratamiento antihipertensivo reduce significativamente el riesgo de eventos cardiovasculares mayores como accidentes cerebrovasculares o enfermedad coronaria. Sin embargo, la falta de adherencia al tratamiento es un problema frecuente en el control de la hipertensión arterial. Países como Inglaterra han reportado un $20 \%$ de abandono del tratamiento antihipertensivo a los seis meses de iniciada la terapia y de $28 \%$ al año ${ }^{(16)}$, en Estados Unidos se evidenció que sólo el $35 \%$ de los pacientes hipertensos permanecían adheridos a su tratamiento luego de un año de iniciado y el $28 \%$ descontinuaba la terapia ${ }^{(11)}$. En Perú se ha reportado que sólo el $39,5 \%$ de los pacientes hipertensos reciben tratamiento y únicamente el $14 \%$ de ellos tienen la presión arterial controlada ${ }^{(17)}$.

En nuestro estudio, los factores asociados a no-adherencia de tratamiento fueron sexo masculino (ORa: 0,45, IC95\% 0,201,04), trabajador independiente (ORa:3,88, IC95\% 1,519,97), IMC mayor de 30 (ORa:0,23, IC95\% 0,07-0,70). La falta de adherencia o cumplimiento inadecuado del tratamiento disminuye la efectividad del tratamiento antihipertensivo. Una terapia constante en el tiempo controla mejor la presión arterial, disminuye el número de hospitalizaciones y reduce los costos sanitarios ${ }^{(6,15,18)}$. Se han identificado diversos factores asociados a la falta de adherencia al tratamiento antihipertensivo; estos factores pueden clasificarse de acuerdo con el modelo multidimensional planteado por la Organización Mundial de la Salud (relacionados al paciente, factores socioeconómicos, comorbilidades, relacionados a la terapia, y relacionados al sistema de salud). Dentro del grupo de Factores Relacionados al Paciente destacan: 1) Índice de masa corporal elevado, identificaron en una cohorte que la obesidad se comporta como un predictor de no adherencia, del mismo modo identificaron que hombres con sobrepeso u obesidad tienen mayor riesgo de no adherencia a la terapia antihipertensiva ${ }^{(10,14)}$; 2) Escaso conocimiento sobre su enfermedad $^{\left({ }^{(1)}\right)}$ : el pobre conocimiento sobre la enfermedad y tratamiento de la hipertensión disminuye la adherencia al tratamiento. Dentro de los Factores socioeconómicos que disminuyen la adherencia al tratamiento destacan: 1) Sexo masculino ${ }^{(10,20)}$; 2) Edad adulta joven ${ }^{(10,13,21-23)}$; 3) Raza latina o afroamericana $^{(14)}$; 4) No tener pareja estable ${ }^{(24)}$; 5) Menor nivel de educación ${ }^{(12,25,26)}$; 6) Ingreso económico bajo ${ }^{(14)}$; 7) Primas de salud elevadas ${ }^{(15)}$. Dentro de los antecedentes de salud que disminuyen la adherencia al tratamiento está contar con una o más comorbilidades en adición a la hipertensión ${ }^{(14)}$. Los factores relacionados a la terapia farmacológica que disminuyen la adherencia al tratamiento antihipertensivo son: 1) Reacción adversa al medicamento $^{(19,26)}$; 2) Regímenes de tratamiento con numerosos tipos de medicamentos ${ }^{(27)}$, 3) Regímenes con múltiples dosis de medicamentos ${ }^{(9,28)}$.

Los hallazgos de nuestra investigación deben ser entendidos bajo las siguientes condiciones. La pobre adherencia es considerada un problema identificable y prevenible ${ }^{(29)}$. Para ello se han establecido varias escalas que permiten medir la adherencia al tratamiento de forma directa o indirecta.
Dentro de los métodos directos destacan el monitoreo electrónico, el conteo de píldora y el dosaje directo de la concentración del medicamento en sangre. Los métodos indirectos incluyen instrumentos como la escala de Adherencia Morisky ${ }^{(30)}$, la escala de Krosuel-Wood ${ }^{(31)}$, la escala de Hill-Bone ${ }^{(32)}$ y los relacionados a la dispensación por farmacia (proporción de posesión de medicación y proporción de días cubiertos). Nuestro estudio utilizó métodos indirectos como tamizaje para identificar pacientes no adheridos al tratamiento antihipertensivo; los cuales una vez identificados, los autores sugerirían implementar métodos directos para supervisión estrecha de adherencia al tratamiento.

En los pacientes con diagnóstico de hipertensión esencial existen factores de riesgo modificables y no modificables asociados a la no-adherencia al tratamiento. Se deben considerar estos factores para implementar estrategias de tamizaje y focalizar las intervenciones para adherir a los pacientes renuentes a su tratamiento. Los resultados del presente estudio son compatibles con relación a los hallazgos de otros estudios, pero llama la atención que la condición de trabajador independiente se asocia a no-adherencia al tratamiento farmacológico de la HTA.

\section{REFERENCIAS BIBLIOGRÁFICAS}

1. Pagès-Puigdemont N, Mangues MA, Masip M, Gabriele G, FernándezMaldonado L, Blancafort S, et al. Patients' Perspective of Medication Adherence in Chronic Conditions: A Qualitative Study. Adv Ther. 2016;33(10):1740-54. doi:10.1007/s12325-016-0394-6

2. Wolf-Maier K, Cooper RS, Kramer H, Banegas JR, Giampaoli S, Joffres $M R$, et al. Hypertension treatment and control in five European countries, Canada, and the United States. Hypertension. 2004;43(1):10-7. doi:10.1161/01.HYP.0000103630.72812.10

3. Tedla YG, Bautista LE. Drug Side Effect Symptoms and Adherence to Antihypertensive Medication. Am J Hypertens. 2016;29(6):772-9. doi:10.1093/ajh/hpv185

4. Hill MN, Miller NH, Degeest S, American Society of Hypertension Writing Group, Materson BJ, Black HR, et al. Adherence and persistence with taking medication to control high blood pressure. J Am Soc Hypertens. 2011;5(1):56-63. doi:10.1016/j.jash.2011.01.001

5. Mazzaglia G, Ambrosioni E, Alacqua M, Filippi A, Sessa E, Immordino V, et al. Adherence to antihypertensive medications and cardiovascular morbidity among newly diagnosed hypertensive patients. Circulation. $2009 ; 120(16): 1598-605$. doi:10.1161/CIRCULATIONAHA.108.830299

6. Corrao G, Parodi A, Nicotra F, Zambon A, Merlino L, Cesana G, et al. Better compliance to antihypertensive medications reduces cardiovascular risk. J Hypertens. 2011;29(3):610-8. doi:10.1097/HJH.0b013e328342ca97

7. Grassi G, Seravalle G, Mancia G. Cardiovascular consequences of poor compliance to antihypertensive therapy. Blood Press. 2011;20(4):196-203. doi:10.3109/08037051.2011.557902

8. Burnier M, Egan BM. Adherence in Hypertension. Circulation $\mathrm{R}$ e s e a r ch. $\quad 2019 ; 124(7): 1124-40$. doi:10.1161/CIRCRESAHA.118.313220

9. Cutler DM, Everett W. Thinking outside the pillbox--medication adherence as a priority for health care reform. N Engl J Med. 2010;362(17):1553-5. doi:10.1056/NEJMp1002305

10. Bailey JE, Hajjar M, Shoib B, Tang J, Ray MM, Wan JY. Risk factors associated with antihypertensive medication nonadherence in a statewide Medicaid population. Am J Med Sci. 2014;348(5):410-5. doi:10.1097/MAJ.0b013e31825ce50f

11. Hyre AD, Krousel-Wood MA, Muntner P, Kawasaki L, DeSalvo KB. Prevalence and predictors of poor antihypertensive medication adherence in an urban health clinic setting. J Clin Hypertens (Greenwich). 2007;9(3):179-86. doi:10.1111/j.15246175.2007.06372.x

12. Cuffee YL, Hargraves JL, Rosal M, Briesacher BA, Schoenthaler A, Person $\mathrm{S}$, et al. Reported racial discrimination, trust in physicians, 
and medication adherence among inner-city African Americans with hypertension. Am J Public Health. 2013;103(11):e55-62. doi:10.2105/AJPH.2013.301554

13. Natarajan N, Putnam W, Van Aarsen K, Beverley Lawson K, Burge F. Adherence to antihypertensive medications among family practice patients with diabetes mellitus and hypertension. Can Fam Physician. 2013;59(2):e93-100.

14. van der Laan DM, Elders PJM, Boons CCLM, Beckeringh JJ, Nijpels G Hugtenburg JG. Factors associated with antihypertensive medication non-adherence: a systematic review. J Hum Hypertens. 2017;31(11):687-94. doi:10.1038/jhh.2017.48

15. Taira DA, Wong KS, Frech-Tamas F, Chung RS. Copayment level and compliance with antihypertensive medication: analysis and policy implications for managed care. Am J Manag Care. 2006;12(11):678-83.

16. Burke TA, Sturkenboom MC, Lu S, Wentworth CE, Lin Y, Rhoads GG. Discontinuation of antihypertensive drugs among newly diagnosed hypertensive patients in UK general practice. J Hypertens. 2006;24(6):1193-200. doi:10.1097/01.hjh.0000226211.95936.f5

17. Medina-Lezama J, Zea-Diaz H, Morey-Vargas OL, Bolaños-Salazar JF, Postigo-Macdowall M, Paredes-Díaz S, et al. Prevalence and patterns of hypertension in Peruvian Andean Hispanics: the PREVENCION study. J A m Soc Hypertens. $2007 ; 1$ (3):216-25. doi:10.1016/j.jash.2007.02.003

18. Kim S, Shin DW, Yun JM, Hwang Y, Park SK, Ko Y-J, et al. Medication Adherence and the Risk of Cardiovascular Mortality and Hospitalization Among Patients With Newly Prescribed Antihypertensive Medications. Hypertension. 2016;67(3):506-12 doi:10.1161/HYPERTENSIONAHA.115.06731

19. Kim $\mathrm{E}-\mathrm{Y}$, Han $\mathrm{H}-\mathrm{R}$, Jeong $\mathrm{S}$, Kim KB, Park $\mathrm{H}$, Kang $\mathrm{E}$, et al. Does knowledge matter?: intentional medication nonadherence among middle-aged Korean Americans with high blood pressure. J Cardiovasc Nurs. 2007;22(5):397-404. doi:10.1097/01.JCN.0000287038.23186.bd

20. Holt E, Joyce C, Dornelles A, Morisky D, Webber LS, Muntner P, et al. Sex differences in barriers to antihypertensive medication adherence: findings from the cohort study of medication adherence among older adults. J Am Geriatr Soc. 2013;61(4):558-64 doi:10.1111/jgs.12171

21. Morris AB, Li J, Kroenke K, Bruner-England TE, Young JM, Murray MD. Factors associated with drug adherence and blood pressure control in patients with hypertension. Pharmacotherapy. 2006;26(4):483-92. doi:10.1592/phco.26.4.483

22. Ren XS, Kazis LE, Lee A, Zhang H, Miller DR. Identifying patient and physician characteristics that affect compliance with antihypertensive medications. J Clin Pharm Ther. 2002;27(1):47-56. doi:10.1046/j.1365-2710.2002.00387.x

23. Lötsch F, Auer-Hackenberg L, Groger M, Rehman K, Morrison V, Holmes $\mathrm{E}$, et al. Adherence of patients to long-term medication: a cross-sectional study of antihypertensive regimens in Austria. Wien Klin Wochenschr. 2015;127(9-10):379-84. doi:10.1007/s00508-0150782-y

24. Trivedi RB, Ayotte B, Edelman D, Bosworth HB. The association of emotional well-being and marital status with treatment adherence among patients with hypertension. J Behav Med. 2008;31(6):489-97. doi:10.1007/s10865-008-9173-4

25. Crowley MJ, Grubber JM, Olsen MK, Bosworth HB. Factors associated with non-adherence to three hypertension self-management behaviors: preliminary data for a new instrument. J Gen Intern Med. 2013;28(1):99-106. doi:10.1007/s11606-012-2195-1

26. Lowry KP, Dudley TK, Oddone EZ, Bosworth HB. Intentional and unintentional nonadherence to antihypertensive medication. Ann Pharmacother. 2005;39(7-8):1198-203. doi:10.1345/aph.1E594

27. Xie L, Frech-Tamas F, Marrett E, Baser O. A medication adherence and persistence comparison of hypertensive patients treated with single, double- and triple-pill combination therapy. Curr Med Res Opin. 2014;30(12):2415-22. doi:10.1185/03007995.2014.964853

28. Zhang Z-Y, Yu Y-L, Asayama K, Hansen TW, Maestre GE, Staessen JA Starting Antihypertensive Drug Treatment With Combination Therapy: Controversies in Hypertension - Con Side of the Argument. $\mathrm{H}$ y p e rte n s i o n . $2021 ; 77$ ( 3 ): $788-98$. doi:10.1161/HYPERTENSIONAHA.120.12858

29. Poulter NR, Borghi C, Parati G, Pathak A, Toli D, Williams B, et al. Medication adherence in hypertension. J Hypertens. 2020;38(4):579-87. doi:10.1097/HJH.0000000000002294

30. Morisky DE, Ang A, Krousel-Wood M, Ward HJ. Predictive validity of a medication adherence measure in an outpatient setting. J Clin Hypertens (Greenwich). 2008;10(5):348-54. doi:10.1111/j.1751. 7176.2008.07572.x

31. Krousel-Wood M, Joyce C, Holt EW, Levitan EB, Dornelles A, Webber LS, et al. Development and evaluation of a self-report tool to predict low pharmacy refill adherence in elderly patients with uncontrolled hypertension. Pharmacotherapy. 2013;33(8):798-811. doi:10.1002/phar.1275

32. Kim MT, Hill MN, Bone LR, Levine DM. Development and testing of the Hill-Bone Compliance to High Blood Pressure Therapy Scale. Prog Cardiovasc Nurs. 2000;15(3):90-6. doi:10.1111/j.1751. 7117.2000.tb00211.x 